\title{
The unified Skyrmion profiles and Static Properties of Nucleons
}

\author{
Duo-Jie Jia*, Xiao-Wei Wang \\ Institute of Theoretical Physics, College of Physics and Electronic \\ Engineering, Northwest Normal University, Lanzhou 730070, P.R. China
}

\begin{abstract}
An unified approximated solution for symmetric Skyrmions was proposed for the SU(2) Skyrme model for baryon numbers up to 8, which take the hybrid form of a kink-like solution and that given by the instanton method. The Skyrmion profiles are examined by 'computing lowest soliton energy as well as the static properties of nucleons within the framework of collective quantization, with a good agreement with the exact numeric results. The comparisons with the previous computations as well as the experimental data are also given.
\end{abstract}

Keywords: Skyrme Model, Soliton, Nucleons

'PACS: 12.38.-t, 11.15.Tk, 12.38.Aw

\section{Introduction}

Due to its substantial connections with the baryon phenomenology, the Skyrme model[1], which appears as the effective field theory of QCD at low-energy limit, has been visited extensively since 1980's (see, [2], for a review), of which the soliton solution, known as Skyrmion, has been often used to explore the baryon and barynic system spectrum and their static properties, particularly, in the case of the small baryon numbers $(B)[3,4,5,6,7]$. Owing to the high nonlinearity, the most of Skyrmion studies was confined to the numerical 'approach [9, 10, 3, 4, 7]. It is still worthwhile, however, to seek the approximated analytic soluitions of Skyrmions consid'ering its extensive applications in baryon phenomenology and nucler physics. The some of efforts have been made in finding the approximated solution to the massless Skyrme model $[11,6,12,13,20]$, but few has been done to the massive Skyrme model, except for the exploration by Manton and Atiyah [17].

In this Letter, we revisit the static solutions of Symmetric Skyrmions in the $S U(2)$ massive Skyrme model, which includes the chiral symmetry breaking term (namely, pion mass term). An analtyical Skyrmion profile is proposed for the massive Skyrme mode with the baryon number up to 8 by writing it as the hybrid form of a kink-like solution and the analytic solution obtained by the instanton method [6]. We show that this profile is in well consistent with the exact numeric solution obtained via the standard relaxation method. To determine the parameters involved in the profile, the downhill simplex algorithm is applied in lowering the energy as best as possible. The static properties of nucleon as well as delta are then computed using the $B=1$ solution in the case of the reduced pion mass

\footnotetext{
${ }^{*}$ Corresponding author

Email address: jiadj@nwnu. edu.cn (Duo-Jie Jia*)
}

$m=0.526$ within the framework of the semiclassical quantization of the Skyrme model, and compared to the previous computations and experimental data.

\section{The analytical profiles for Skyrmions}

The $S U(2)$ Skyrme model [1] with pion mass term is given by

$$
\begin{array}{r}
L=\int d^{3} x\left\{\frac{f_{\pi}^{2}}{4} \operatorname{tr}\left(\partial_{\mu} U \partial^{\mu} U^{\dagger}\right)+\frac{1}{32 e^{2}} \operatorname{tr}\left[\partial_{\mu} U U^{\dagger}, \partial_{\nu} U U^{\dagger}\right]^{2}\right. \\
\left.+\frac{m_{\pi}^{2}}{2} f_{\pi}^{2} \operatorname{tr}(U-1)\right\}
\end{array}
$$

with $U(x, t) \in S U(2)$ the nonlinear realization of the chiral field describing the $\sigma$ field and $\pi$ mesons under the constrain $U^{\dagger} U=1,2 f_{\pi}$ is the pion decay constant, and $e$ is the dimensionless constant characterizing nonlinear coupling. The last (mass) term in (1) corresponds to the chiral symmetry breaking and ignored in the chirally symmetric Skyrme model. With the weak- $\pi$ expansion

$$
U=(\sigma+i \vec{\tau} \cdot \vec{\pi}) / f_{\pi} \approx 1+\frac{i}{f_{\pi}} \vec{\tau} \cdot \vec{\pi},
$$

( $\vec{\tau}$ are the three Pauli matrices) the model (1) becomes the dynamics of pian

$$
\mathcal{L}^{\pi}=\frac{1}{2}\left(\partial_{\mu} \vec{\pi}\right)^{2}-\frac{m_{\pi}^{2}}{2} \vec{\pi}^{2}+O\left(\pi^{4}\right),
$$

in which the pion-mass term arises from the mass (third) term in (1). The Hamiltonian with respect to the Lagrangian (1) is

$$
\begin{array}{r}
\mathcal{H}=\frac{f_{\pi}}{2 e}\left\{\frac{1}{2} \operatorname{tr}\left(\partial^{i} U \partial^{i} U^{\dagger}\right)-\frac{1}{16} \operatorname{tr}\left[\partial^{i} U U^{\dagger}, \partial^{j} U U^{\dagger}\right]^{2}\right. \\
\left.+m^{2} \operatorname{tr}(1-U)\right\},
\end{array}
$$


with $m=m_{\pi} /\left(e f_{\pi}\right)$ the reduced pian mass (in the unit of $e f_{\pi}$ ), and $\partial^{i}$ are the partial derivatives in the length unit of $1 / e f_{\pi}$.

We use the parameterization of the rational-map ansatz described in [15],

$$
U(r, z)=\exp \left[i F(r) \hat{n}_{R(z)} \cdot \vec{\tau}\right]
$$

where $\hat{n}_{R(z)}$ represents a map from the Riemann spherical coordinate $z=e^{i \varphi} \tan (\theta / 2)$, which is related to the angular coordinate $(\theta, \varphi)$ via the stereographic projection, to the unit iso-triplet through the next stereographic projection in the internal space,

$$
\hat{n}_{R(z)}=\frac{1}{1+|R|^{2}}\left(2 \operatorname{Re} R, 2 \operatorname{Im} R, 1-|R|^{2}\right) .
$$

Here, the map $R(z)$ from a complex plane to $S^{2}=\mathbf{C} \cup\{\infty\}$ in the internal space has also to be a holomorphic map of degree $B$. This implies that $R(z)$ can be written as a ratio of two polynomials $R(z)=p(z) / q(z)$, where $p$ and $q$ are polynomials in $z$ such that $\max \{\operatorname{deg}(p), \operatorname{deg}(q)\}=B$, and $p$ and $q$ have no common factors. In (3), $F(r)$ is a real profile function of the radial coordinate $r$, which has to satisfy the boundary condition $F(0)=\pi, F(\infty)=0$. When (3) and (4) applied, $U$ becomes

$$
U(r, z)=\frac{1}{1+|R|^{2}}\left[\begin{array}{cc}
e^{i F}+|R|^{2} e^{-i F} & 2 i R^{*} \sin (F) \\
2 i R \sin (F) & e^{-i F}+|R|^{2} e^{i F}
\end{array}\right]
$$

With (5) and (2), one has for the static energy for (1)

$$
\begin{gathered}
M^{S K}=6 \pi^{2} \frac{f_{\pi}}{e} E \\
E=\frac{1}{3 \pi} \int_{0}^{\infty} d x x^{2}\left[F_{x}^{2}+\frac{2}{x^{2}} B \sin ^{2}(F)\left(1+F_{x}^{2}\right)\right. \\
\left.+\mathcal{I} \frac{\sin ^{4}(F)}{x^{4}}+2 m^{2}(1-\cos F)\right]
\end{gathered}
$$

where

$$
\mathcal{I}=\frac{1}{4 \pi} \int\left(\frac{1+|z|^{2}}{1+|R|^{2}}\left|\frac{d R}{d z}\right|\right)^{4} \frac{2 i d z d z^{*}}{\left(1+|z|^{2}\right)^{2}}
$$

Here, the dimensionless variable $x=e f_{\pi} r$ and $F_{x} \equiv d F(x) / d x$ are used. The equation of motion of (7) is

$$
\begin{aligned}
(1+ & \left.2 B \frac{\sin ^{2} F}{x^{2}}\right) F_{x x}+\frac{2}{x} F_{x}+\frac{\sin (2 F)}{x^{2}} \\
& \times\left(F_{x}^{2}-1-I \frac{\sin ^{2} F}{x^{2}}\right)=m^{2} \sin F,
\end{aligned}
$$

The numerical solutions to (9) and to the equation of motion of the original functional (2) via the full field $U$ calculation are both presented by Battye, Sutcliffe and others [3]. It is shown there that an excellent agreement was achieved between the solutions given by rational map approximation and full field calculation for the baryon number $B$ up to 12 . Here, we reexamine only the rational map solutions to the Skyrmions with $B=1 \sim 8$ for the case of the reduced pion mass $m=0.526$, which is of the physically interest [10], by solving (9) analytically and numerically.
For given $B$, the minimization of the energy (7) should be carried out in two steps: to minimize $\mathcal{I}(8)$ as a functional of $R(z)$ at first and then to minimize energy (7) for the optimized value of $\mathcal{I}$. The first step is done in [21] and we simply list optimized values of $\mathcal{I}$ in Table I as a input for our calculation.

The Skyrmion profile ansatz we propose is

$$
\begin{array}{r}
F(x)=4 w \arctan [\exp (-c x)]+\pi(1-w) \\
\times\left[1-\left(\frac{\sinh ^{2}(d m x)}{a^{2}+\sinh ^{2}(d m x)}\right)^{1 / 2}\right]
\end{array}
$$

where $m \neq 0$, and $(a, c, d, w)$ are the parameters to be determined variationally. We note that the the second term in (10) appears as the hyperbolic Skyrmion form obtained using instanton holonomy [16, 17] where $a$ is related to the instanton scale $\lambda$ through

$$
a=\frac{2 \lambda}{\left(1+\lambda^{2}\right)} .
$$

Given $F(x)$ specified by (10), we are able to minimize (7) with respect to the variational parameters $(a, c, d, w)$ for $B=$ $1, \cdots, 8$ using the downhill simplex method (the Neilder-Mead algorithm). The optimized parameters are listed in Table I, including the soliton energy per $B$ calculated accordingly and the corresponding numeric results obtained via the relaxation procedure.

The profile solution (10), with the parameters in Table I, is plotted in Fig.1 and compared to the numerical solution to the equation (9). To the later, we employ the relaxation algorithm with the different initial setups of $F(x)$, ranging from the Gaussian distribution, to the kink configuration, and find that the same result is achieved. It can be seen from Table I and Fig.1 that a quite well agreement is achieved between the numerical solutions and that given by (10).

We also present the fit of the parameters depending upon $B$, which are given by

$$
\begin{aligned}
& \lambda=0.4639+1.488 \exp (15.038-15.361 B) \\
& -0.5466 \exp (1.7442-1.7816 B) \\
& c=0.7196+2.2871 \frac{B-0.94845}{B-105.59} \exp \left\{-\left[\ln \left(\frac{B}{0.2236}\right)\right]^{2} / 6.774\right\} \\
& d=0.7981 B \\
& w=251.4-\frac{248.8}{1+\exp \left\{21.394 \frac{B-0.46709}{B+0.066519}-20.404\right\}},
\end{aligned}
$$

and shown in Fig.3.

For the dependence of the charge one Skyrmion profile on the reduced pion mass $m$, we examine (10) for the different $m=1,2,3$, respectively. In Table II, the list is given for the parameters in (10), which are calculated with the same procedure as before, and for the corresponding soliton energies obtaind by (10) (marked by Ana) and the energies obtained numerically (marked by Num). The chiral angle profiles obtained analytically and numerically are both plotted in Fig. 2 for $m=0,1,2,3$, where the Skyrmion profile for the massless model is otained in [20] and used simply here for $m=0$ case. The results here show that the function (10) for $B \geq 2$ can be taken to be the approximated analytical solution to the 
Skyrme model, which is presented in an analytical form for any low $B$. Since (10) are not the most general expression of $F(x)$, the variation of the parameters does not necessarily lead to a real analytical solution. However, considering that exact analytical solution is difficult (almost impossible) to be found in most cases of the highly nonlinear problems like the Skyrme model, the solutions presented here can be an alternative to the exact analytical solution in so far as we are going to search the approximated solution which approaches the real solution as better as possible.

\section{The static properties of nucleons}

It is known that the static properties of nucleons can be extracted by semi-classically quantizing the spinning modes of Skyrme Lagrangian using the collective variables [9, 10]. Following Adkin et al. [10], we use the charge one solution of (10) to compute the static properties of nucleons and nucleonisobar $(\Delta)$ within the framework of the bosonic quantization of Skyrme model.

Choosing a $S U(2)$-variable $A(t)$ as the collective variables, and substituting $U=A(t) U_{0}(x) A(t)^{\dagger}$ into (1), one can show that the Hamiltonian for the bosonically quantizatized model has an eigenvalue $\langle H\rangle=M^{S K}+J(J+1) /\left(2 I_{0} \Lambda\right)$ with $M^{S K}$ the soliton energy for the static hedgehog $\operatorname{Skyrmion}\left(\hat{n}_{R(z)}=\hat{r}\right)$ for which $\mathcal{I}=1, I_{0}=\pi /\left(3 e^{3} f_{\pi}\right)$, and

$$
\Lambda=8 \int_{0}^{\infty} x^{2} d x \sin ^{2} F\left[1+F_{x}^{2}+\sin ^{2} F / x^{2}\right] .
$$

Hence, the masses of the nucleon and $\Delta$-isobar are given by (with $J=1 / 2$ and $3 / 2$ )

$$
\begin{aligned}
& M_{N}=M^{S K}+\frac{3}{8 I_{0} \Lambda}, \\
& M_{\Delta}=M^{S K}+\frac{15}{8 I_{0} \Lambda} .
\end{aligned}
$$

As done in [10], we choose to adjust model parameters $\left(f_{\pi}, e\right)$ to fit the hadron masses, namely, the $N, \Delta$, and $\pi$ masses through (14), where the other quantities involved are given by (7) and (13). Our reports for the computation of the static properties of baryons are presented in Table III, including the experimental data as well as the results in Ref.[9, 10]. The computations parallel the computation reported in [10]. We also list the most relevant formulas for our computations, for instance, the isoscalor root mean square(r.m.s) radius and isoscalor magnetic r.m.s radius

$$
\begin{aligned}
e f_{\pi}\left\langle r^{2}\right\rangle_{I=0}^{1 / 2} & =\left\{-\frac{2}{\pi} \int_{0}^{\infty} x^{2} \sin ^{2} F F_{x}\right\}^{1 / 2} \\
e f_{\pi}\left\langle r^{2}\right\rangle_{M, I=0}^{1 / 2} & =\left\{\frac{\int_{0}^{\infty} x^{4} \sin ^{2} F F_{x} d x}{\int_{0}^{\infty} x^{2} \sin ^{2} F F_{x} d x}\right\}^{1 / 2}
\end{aligned}
$$

and the magnetic moments for proton and neutron

$$
\mu_{p, n}=\mu_{p, n}^{I=0}+\mu_{p, n}^{I=1}=\frac{\left\langle r^{2}\right\rangle_{I=0}}{9} M_{N}\left(M_{\Delta}-M_{N}\right) \pm \frac{M_{N}}{2\left(M_{\Delta}-M_{N}\right)},
$$

where plus and minus correspond to proton and neutron, respectively. Since the chiral symmetry is broken explicitly, the isovector electric and magnetic charge radii are finite and can be calculated by

$$
\begin{aligned}
e^{2} f_{\pi}^{2}\left\langle r^{2}\right\rangle_{I=1} & =\int_{0}^{\infty} x^{2} \rho_{I=1}(x) d x \\
e^{2} f_{\pi}^{2}\left\langle r^{2}\right\rangle_{M, I=1} & =\int_{0}^{\infty} x^{2} \rho_{M, I=1}(x) d x
\end{aligned}
$$

where $\rho_{I=1}(x)$ is the normalized electric iso-vector charge distributions and can be derived from the zero component of the vector current. It can be shown that these two densities are identically same and can be given by

$$
\rho_{I=1}(x)=\rho_{M, I=1}(x)=\frac{x^{2} \sin ^{2}(F)\left\{1+F_{x}^{2}+\sin ^{2}(F) / x^{2}\right\}}{\int_{0}^{\infty} x^{2} \sin ^{2}(F)\left\{1+F_{x}^{2}+\sin ^{2}(F) / x^{2}\right\} d x} .
$$

Therefore, the r.m.s radii of the isovector electric and magnetic charge are happen to be same.

For the nuclear interaction, the pian-nucleon sigma term can be given by the matrix element in the nucleon states $N(p)$ [18], which in our case becomes (see [10])

$\sigma=\frac{1}{2} f_{\pi}^{2} m_{\pi}^{2} \int_{0}^{\infty} d V\left[2-\operatorname{tr} U_{0}\right]=\frac{4 \pi m_{\pi}^{2}}{e^{3} f_{\pi}} \int_{0}^{\infty} x^{2} d x(1-\cos (F))$,

where $U_{0}$ is the the soliton solution of the chiral symmetric theory(namely, the massless theory). The result is $\sigma=38 \mathrm{MeV}$, as given in [10]. The calculated result for sigma term here is found to be $38.099 \mathrm{MeV}$ and listed in Table III, for which $U_{0}$ is the the soliton solution of the chirally symmetry-breaking theory (namely, the massive theory (1)). Being connected with the amplitude for meson-nucleon scattering, the sigma term can be extracted from the experimental data, with the "world average" estimate $36 \pm 20 \mathrm{MeV}$ [18, 19], and is also listed in the table.

\section{Concluding remarks}

We show that the hybrid form of a kink-like solution and the approximated Skyrmion profile given by the instanton method are suited to approximate the exact profile for the symmetric Skyrmions in the Skyrme model with pion mass term for all baryon numbers up $B$ to 8 . This is first unified expression for the symmetric Skyrmions for the SU(2) Skyrme model with chiral symmetry broken. The computation of the corresponding soliton energies, and the static properties of nucleons within the framework of collective quantization, shows that the yielded Skyrmion profiles here are all in a good agreement with that given by the exact numeric solution.

\section{Acknowledgements}

D. J thanks Q. Wang and ChuengRyong Ji for discussions. This work is supported in part by the National Natural Science Foundation of China (No.10965005) and The Projectsponsored by SRF for ROCS, SEM. 


\section{References}

[1] T.H.R. Skyrme, Nucl. Phys. 31(1961)556.

[2] I. Zahed and G. Brown, Phys. Rept. 142(1986)1.

[3] P.M. Sutcliffe, Phys. Lett. B 292(1992)104.

[4] R. Battye and P.M. Sutcliffe, Phys. Rev. Lett.79(1997)363.

[5] M.F. Atiyah and N.S. Manton, Commun. Math. Phys. 152(1993)391.

[6] M.F. Atiyah and N.S. Manton, Phys. Lett. B222(1989)438.

[7] R. Battye and P.M. Sutcliffe, Phys. Rev. C73 (2006)055205.

[8] C. Adam, J.S. Sánchez-Guillén, and A.Wereszczynski, arXiv:1001. 4544[hep-th]

[9] G.S. Adkins, C.R. Nappi and E. Witten, Nucl. Phys. B228(1983)552.

[10] G.S. Adkins and C.R. Nappi, Nucl. Phys. B 233(1984)109.

[11] J. Ananias et al., J. Math. Phys. 32, 7(1991)1949.

[12] J.A. Ponciano et al., Phys. Rev. C64(2001)045205.

[13] J. Yamashita and M. Hirayama, Phys. Lett. B 642(2006)160.

[14] L.D. Faddeev, Lett. Math. Phys. 1(1976) 289.

[15] C.J.Houghton,N.S.Manton and P.M.Sutcliffe,Nucl.Phys.B 510(1998) 507.

[16] N.S.Manton,T.M.Samols,J.Phys.A23(1990)3749.

[17] M.F. Atiyah and P.M. Sutcliffe, Phys. Lett. B 605 (2005) 106-11

[18] H. Pagels, Phys. Rept.16 (1975)219.

[19] E. Reya, Rev. Mod. Phys. 46 (1974)7159.

[20] Duo-jie Jia, Xiao-wei Wang and Feng Liu, Submitted to Chin. Phys. Lett. arXiv:0912.5142].

[21] R.A.Battye,P.M.Sutcliffe,Rev.Math.Phys. 14(2002)29. 


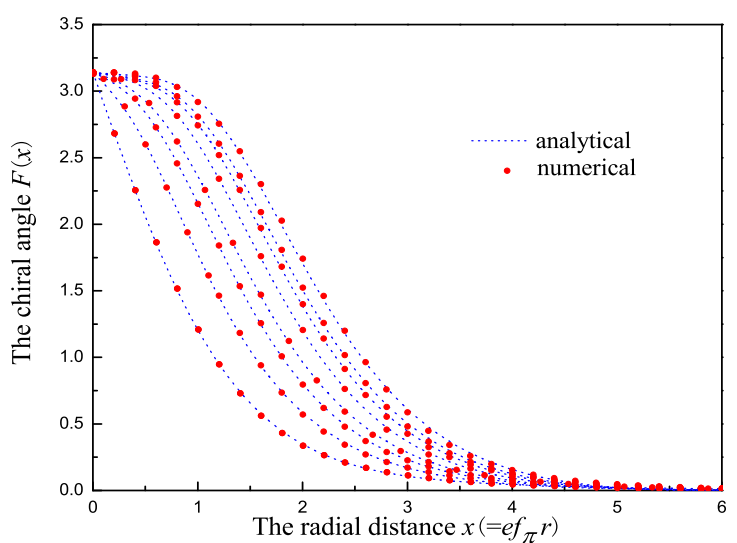

Fig. 1.The chiral angle profile $F(x)$ v.s. the radial coordinate for baryon numbers from 1 (the most bottom) to 8 (the most top) and for the pion mass $\mathrm{m}=0.526$.

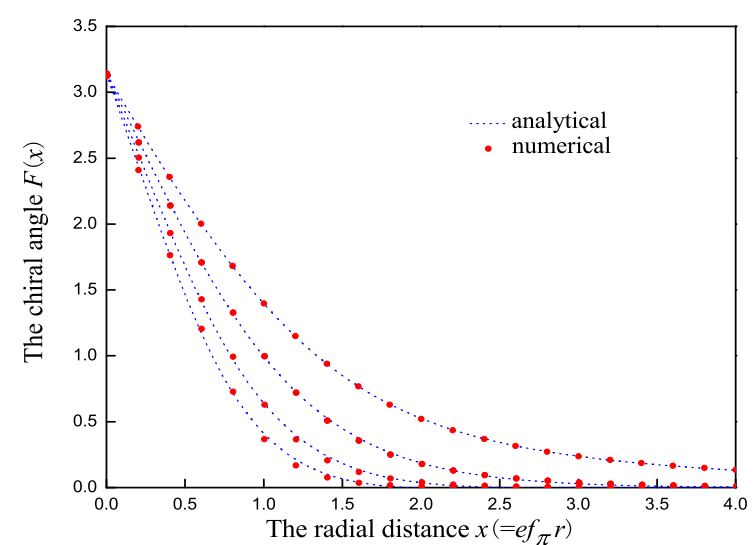

Fig. 2.The chiral angle profile $F(x)$ v.s. the radial coordinate for the pion mass from 0 (the most top) to 3 (the most bottom) and for baryon number $\mathrm{B}=1$.

Table I

\begin{tabular}{|c|c|c|c|c|c|c|c|c|}
\hline$B$ & 1 & 2 & 3 & 4 & 5 & 6 & 7 & 8 \\
\hline $\mathcal{I}$ & 1 & 5.81 & 13.58 & 20.65 & 35.75 & 50.76 & 60.87 & 85.63 \\
\hline$\lambda$ & 1.016 & 0.3751 & 0.4508 & 0.4558 & 0.4629 & 0.4651 & 0.4650 & 0.4658 \\
\hline$c$ & 0.8058 & 1.314 & 1.306 & 1.223 & 1.166 & 1.114 & 1.070 & 1.019 \\
\hline$d$ & 0.7981 & 1.309 & 1.237 & 1.147 & 1.093 & 1.043 & 1.001 & 0.9532 \\
\hline$w$ & 3.203 & 3.760 & 20.45 & 34.08 & 58.63 & 77.72 & 85.91 & 102.3 \\
\hline$\frac{E}{B}$ (Ana.) & 1.3084 & 1.2848 & 1.2603 & 1.2078 & 1.2230 & 1.2147 & 1.1817 & 1.1984 \\
\hline$\frac{E}{B}$ (Num.) & 1.3077 & 1.2833 & 1.2593 & 1.2069 & 1.2219 & 1.2132 & 1.1799 & 1.1953 \\
\hline
\end{tabular}




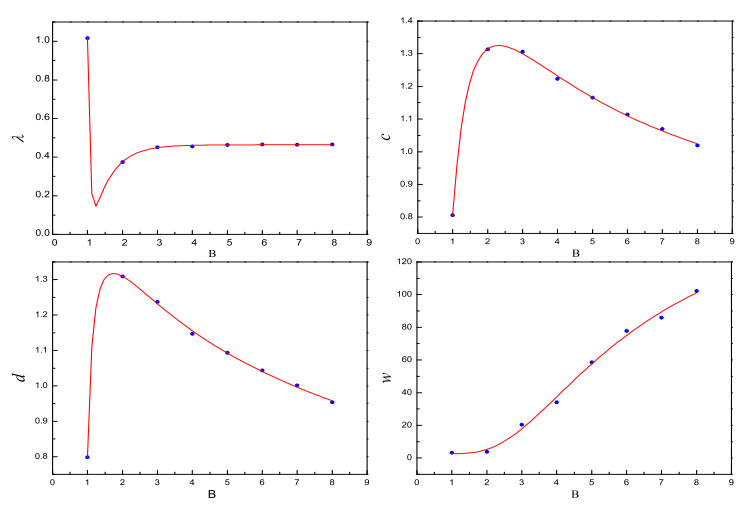

Fig. 3. The values of the parameters $\lambda, c, d, w$ (the solid circles) presented in Table I plotted against B.The solid lines shows the fit of the corresponding parameters.

Table II

\begin{tabular}{|c|c|c|c|}
\hline \multicolumn{4}{|c|}{$\mathrm{B}=1, \mathrm{I}=1$} \\
\hline$m$ & 1 & 2 & 3 \\
\hline$\lambda$ & 1.001 & 0.9990 & 0.9995 \\
\hline$c$ & 1.581 & 1.813 & 2.116 \\
\hline$d$ & 0.8814 & 0.5419 & 0.4236 \\
\hline$w$ & -0.6877 & -1.746 & -2.209 \\
\hline$E$ (Ana.) & 1.418 & 1.647 & 1.855 \\
\hline (Num.) & 1.416 & 1.642 & 1.847 \\
\hline
\end{tabular}

Table III

\begin{tabular}{|c|c|c|c|c|c|}
\hline Quantities & Ref.[9] & Ref.[10] & Analytical & Num. & Expt. \\
\hline$M_{N}(\mathrm{MeV})$ & 938.9(input) & 938.9(input) & 938.9(input) & 938.9(input) & 938.9 \\
\hline$M_{\Delta}(\mathrm{MeV})$ & 1232 (input) & 1232 (input) & 1232(input) & 1232(input) & 1232 \\
\hline$m_{\pi}(\mathrm{MeV})$ & 0 (input) & 138 (input) & 138 (input) & 138 (input) & 138 \\
\hline $2 f_{\pi}(\mathrm{MeV})$ & 129 & 108 & 108.20 & 108.27 & 186 \\
\hline$e$ & 5.45 & 4.84 & 4.8424 & 4.8430 & - \\
\hline$\left\langle r^{2}\right\rangle_{I=0}^{1 / 2}(\mathrm{fm})$ & 0.59 & 0.68 & 0.6825 & 0.6821 & 0.72 \\
\hline$\left\langle r^{2}\right\rangle_{I=1}^{1 / 2}(\mathrm{fm})$ & $\infty$ & 1.04 & 1.0589 & 1.0408 & 0.88 \\
\hline$\left\langle r^{2}\right\rangle_{M, I=0}^{1 / 2}(\mathrm{fm})$ & 0.92 & 0.95 & 0.9619 & 0.9542 & 0.81 \\
\hline$\left\langle r^{2}\right\rangle_{M, I=1}^{1 / 2}(\mathrm{fm})$ & $\infty$ & 1.04 & 1.0589 & 1.0408 & 0.80 \\
\hline$\mu_{p}$ & 1.87 & 1.97 & 1.9669 & 1.9664 & 2.79 \\
\hline$\mu_{n}$ & -1.31 & -1.24 & -1.2365 & -1.2369 & -1.91 \\
\hline$\left|\mu_{p} / \mu_{n}\right|$ & 1.43 & 1.59 & 1.5906 & 1.5898 & 1.46 \\
\hline$\sigma(\mathrm{MeV})$ & - & 38 & 38.099 & 37.726 & $36 \pm 20$ \\
\hline
\end{tabular}

